\title{
Accumulation of the delivered dose based on cone-beam CT and deformable image registration for non-small cell lung cancer treated with hypofractionated radiotherapy
}

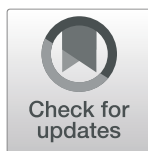

Bin Wang ${ }^{\dagger}$, Da Quan Wang ${ }^{\dagger}$, Mao Sheng Lin, Shi Pei Lu, Jun Zhang, Li Chen, Qi Wen Li, Zhang Kai Cheng, Fang Jie Liu, Jin Yu Guo, Hui Liu* and Bo Qiü

\begin{abstract}
Background: This study aimed to quantify the dosimetric differences between the planned and delivered dose to tumor and normal organs in locally advanced non-small cell lung cancer (LANSCLC) treated with hypofractionated radiotherapy (HRT), and to explore the necessity and identify optimal candidates for adaptive radiotherapy (ART).

Methods: Twenty-seven patients with stage III NSCLC were enrolled. Planned radiation dose was 51Gy in 17 fractions with cone-beam $C T(C B C T)$ acquired at each fraction. Virtual $C T$ was generated by deformable image registration (DIR) of the planning $C T$ to $C B C T$ for dose calculation and accumulation. Dosimetric parameters were compared between original and accumulated plans using Wilcoxon signed rank test. Correlations between dosimetric differences and clinical variables were analyzed using Mann-Whitney $U$ test or Chi-square test.

Results: Patients had varied gross tumor volume (GTV) reduction by HRT (median reduction rate $11.1 \%$, range - 2.944.0\%). The $V_{51}$ of planning target volume for GTV (PTV-GTV) was similar between original and accumulated plans (mean, $88.2 \%$ vs. $87.6 \%, p=0.452$ ). Only $11.1 \%$ of patients had above $5 \%$ relative decrease in $V_{51}$ of PTV-GTV in accumulated plans. Compared to the original plan, limited increase (median relative increase $<5 \%$ ) was observed in doses of total lung (mean dose, $V_{20}$ and $V_{30}$ ), esophagus (mean dose, maximum dose) and heart (mean dose, $V_{30}$ and $V_{40}$ ) in accumulated plans. Less than $30 \%$ of patients had above $5 \%$ relative increase of lung or heart doses. Patients with quick tumor regression or baseline obstructive pneumonitis showed more notable increase in doses to normal structures. Patients with baseline obstructive atelectasis showed notable decrease (10.3\%) in dose coverage of PTV-GTV.
\end{abstract}

Conclusions: LANSCLC patients treated with HRT had sufficient tumor dose coverage and acceptable normal tissue dose deviation. ART should be applied in patients with quick tumor regression and baseline obstructive pneumonitis/atelectasis to spare more normal structures.

Keywords: Non-small cell lung cancer, Hypofractionated radiotherapy, Accumulation, Deformable image registration

\footnotetext{
* Correspondence: liuhuisysucc@sina.com; qiubo@sysucc.org.cn

${ }^{\dagger}$ Bin Wang and Da Quan Wang contributed equally to this work.

Department of Radiation Oncology, Sun Yat-sen University Cancer Center, State Key Laboratory of Oncology in South China, Collaborative Innovation Center for Cancer Medicine, Guangzhou, Guangdong, China
}

(c) The Author(s). 2020 Open Access This article is licensed under a Creative Commons Attribution 4.0 International License, which permits use, sharing, adaptation, distribution and reproduction in any medium or format, as long as you give appropriate credit to the original author(s) and the source, provide a link to the Creative Commons licence, and indicate if changes were made. The images or other third party material in this article are included in the article's Creative Commons licence, unless indicated otherwise in a credit line to the material. If material is not included in the article's Creative Commons licence and your intended use is not permitted by statutory regulation or exceeds the permitted use, you will need to obtain permission directly from the copyright holder. To view a copy of this licence, visit http://creativecommons.org/licenses/by/4.0/ The Creative Commons Public Domain Dedication waiver (http://creativecommons.org/publicdomain/zero/1.0/) applies to the data made available in this article, unless otherwise stated in a credit line to the data. 


\section{Backgorund}

Definitive radiotherapy combined with chemotherapy remains the main part of treatment for patients with unresectable locally advanced non-small cell lung cancer (LANS CLC) $[1,2]$. Hypofractionated radiotherapy (HRT) has been demonstrated as an effective approach for dose escalation $[3,4]$. The use of HRT is still challenging due to the close distance of target and organs at risk (OAR) in LANSCLC. The precise dose delivery has been achieved by the development of radiation technology, such as the simultaneous integrated boost intensity-modulated radiation therapy (SIBIMRT) and on-board imaging guidance. However, it might be compromised by inter-fractional changes, which were caused by position shifts and anatomical changes of tumors and normal structures during treatment [5]. Such effects could be remarkable in HRT due to its higher fraction dose and significant tumor shrinkage [6].

Image-guided adaptive radiotherapy (ART) has been developed to account for the anatomic variations. It has been proposed that ART may be beneficial in reducing toxicities and allowing safe dose escalation in HRT. However, it is not widely used in clinic due to its timeconsuming process and low cost-effectiveness. Therefore, it is essential to quantify the dosimetric differences between delivered and planned dose to tumor as well as OAR during HRT, in order to provide more information for proper patient selection. Cone beam CT (CBCT) has been routinely used for position verification and monitoring anatomical changes during radiotherapy for NSCLC patients [7]. However, due to poor resolution and inaccurate Housfield Unit (HU), the image quality of CBCT is typically insufficient for dose calculation and accumulation [8]. The virtual images can be generated by deforming the planning CT to the CBCT using deformable image registration (DIR), which provide realtime anatomy information with improved image quality [9]. This process enables the evaluation and accumulation of inter-fractional dose. Previous studies have confirmed that DIR is a validated tool for dose recalculation and accumulation among NSCLC patients treated with stereotactic body radiotherapy and conventional fractionated radiotherapy [10-13].

In this study, CBCT images and DIR technique were used to investigate the impact of inter-fractional anatomic changes on the dose delivered to tumor and normal structures in LANSCLC treated with HRT. By comparing the planned and delivered treatment dose, we aimed to explore the necessity and identify optimal candidates for ART.

\section{Methods}

\section{Patients and treatment}

Twenty-seven patients with stage III NSCLC were enrolled from a prospective clinical trial (NCT 02573506).
Patients were immobilized and simulated according to the standard protocol for lung cancer [14]. A simulation four-dimensional CT (4DCT) scan was obtained with 3 $\mathrm{mm}$ thickness slices from the atlas to the second lumbar vertebra level to cover the whole neck and lung. The CT scan was performed with the Real-time Position Management system for respiratory gating (Varian Medical Systems, Palo Alto, CA). The CT images were automatically sorted into 10 phases corresponding to a single respiratory cycle. The maximum intensity projection (MIP) images were reconstructed from the 10 phases of images. The gross tumor volume (GTV) was defined as the visible primary tumor and positive lymph nodes on CT scans, which matched the volume of MIP images to account for respiratory motion. The clinical target volume (CTV) was delineated to include GTV plus a $0.5 \mathrm{~cm}$ margin and involved node regions. The planning target volumes for GTV (PTV-GTV) and CTV (PTV-CTV) were created by a uniform expansion of $0.5 \mathrm{~cm}$ surrounding the GTV and CTV, respectively. Thoracic RT was delivered using SIB-IMRT technique with a total dose of 51 Gy to PTV-GTV and 44.2 Gy to PTV-CTV in 17 daily fractions.

The study was reviewed and approved by Ethics Committee of our center. All patients provided signed informed consent for scientific usage of clinical data.

\section{CBCT acquisition}

Patients underwent daily kilovoltage (kv)-based CBCT scan for set-up correction prior to the delivery of each radiation fraction. The CBCT scans were obtained using an Elekta VersaHD system (Elekta Lts, Crawley, UK). All scans were acquired under free breathing with patients in treatment position, which has accounted for internal movement because of the blurring of respiratory motion. The maximum scan length was $26.7 \mathrm{~cm}$ with a slice thickness of $3 \mathrm{~mm}$. Other scanning parameters included $120 \mathrm{kV}$ tube potential, $40 \mathrm{~mA}$ tuber current, $40 \mathrm{~ms}$ exposure time, 640 frames and $360^{\circ}$ data collection. CBCT images obtained at fractions $1,5,9,13$, and 17 of treatment were selected for analyses in this study (Fig. 1).

\section{Virtual CT with deformable image registration}

The planning CT was set as the reference image and the subsequent CBCTs as secondary images in Velocity software Version 3.2.0 (Velocity Medical Solutions, Atlanta, Georgia, USA). First, two sets of images were manually aligned using bonny structures as reference. Then, rigid registration was performed between planning $\mathrm{CT}$ and daily CBCT. After that, a correction procedure was performed to apply a fade correction and enhance low signal regions of the $\mathrm{CBCT}$ prior to the following registration. DIR was performed using modified B-spline deformable registration with mutual information-based 


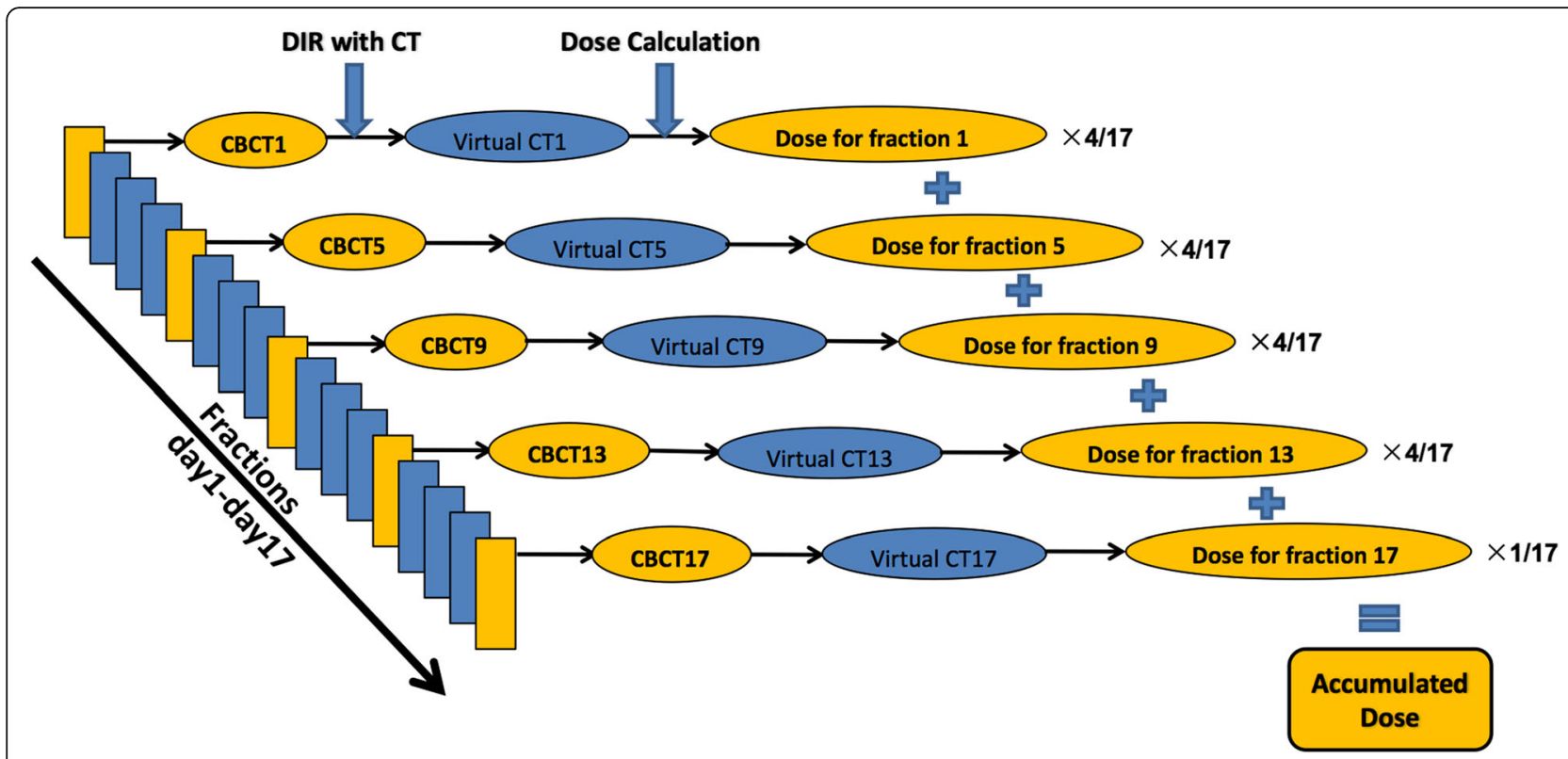

Fig. 1 The study workflow (DIR: deformable image registration)

matching [15]. The deformable vector field was manually reviewed to ensure the quality of DIR. Anatomical landmarks, e.g. bifurcation of airways and great vessels were used to evaluate the DIR accuracy. Once the deformation was reviewed to be acceptable by the operator, a virtual $\mathrm{CT}$ set was generated by deforming the planning CT according to the deformation vector field (DVF). The virtual CT was then exported to the treatment planning system (TPS) for dose calculations.

\section{Dose calculation based on virtual CT}

The treatment beams from the original plan were copied and applied to the isocenters of virtual CT. Doses delivered by the original plan was recalculated on virtual CT images. It was noted that all beam parameters were consistent with the original treatment plan, including isocenter, control points and monitor units.

\section{Dose accumulation}

Delivered dose was calculated for fraction 1, 5, 9, 13 and 17 using the virtual CTs respectively. The calculated dose on the virtual $\mathrm{CT}$ was then deformed back to the planning CT for dose accumulation using DVF obtained in the DIR between planning CT and daily CBCT. The cumulative dose was calculated as follows: $D_{\text {sum }}=$ $\left(D_{\text {frac1 }}+D_{\text {frac5 }}+D_{\text {frac9 }}+D_{\text {frac13 }}\right) \times 4+D_{\text {frac17 }}$. The following dosimetric parameters were recorded from the original and accumulated plan respectively: PTV-GTV: volume receiving $\geq 51 \mathrm{~Gy}\left(\mathrm{~V}_{51}\right)$, dose covering at least 95\% volume $\left(D_{95}\right)$, mean dose; Total lung: mean dose, volume receiving $\geq 20 \mathrm{~Gy} \quad\left(\mathrm{~V}_{20}\right)$ and $30 \mathrm{~Gy} \quad\left(\mathrm{~V}_{30}\right)$;
Esophagus: maximum dose $\left(\mathrm{D}_{\max }\right)$ and mean dose; Heart: mean dose, volume receiving $\geq 30 \mathrm{~Gy}\left(\mathrm{~V}_{30}\right)$ and $40 \mathrm{~Gy}\left(\mathrm{~V}_{40}\right)$.

\section{Statistical analysis}

Dosimetric parameters were presented as mean \pm SD. Relative dosimetric difference between original and accumulated plan was calculated as: (accumulated plan-original plan)/original plan, and was presented as median value with range. In this study, a relative change $\geq 5 \%$ in dosimetric parameters was considered clinically meaningful. Tumor volume and dosimetric parameters were compared between original and accumulated plans using Wilcoxon signed rank test. Correlations between dosimetric differences and clinical variables, including sex, age, tumor location, presence of bulky mediastinal lymph node, cTNM stage, presence of obstructive pneumonitis/atelectasis and tumor regression, were analyzed using Mann-Whitney $U$ test or Chi-square test. All tests were two-sided, and $\mathrm{p}<0.05$ was considered statistically significant. All the analysis was performed with SPSS (Version 22.0).

\section{Results}

\section{Patient characteristics}

The clinical characteristics of 27 patients were summarized in Table 1. Of all patients, the median age at diagnosis was 61 years (range, 42-72). Five cases (18.5\%) were females and $22(81.5 \%)$ were males. Twelve patients (44.4\%) had stage IIIA and 15 (55.6\%) had stage IIIB diseases. There were 22 cases $(81.5 \%)$ with central type tumor and 5 cases (18.5\%) with peripheral type. Baseline obstructive pneumonitis/atelectasis was observed in 11 
Table 1 Patients' clinical characteristics $(n=27)$

\begin{tabular}{ll}
\hline characteristics & $\boldsymbol{n}(\%)$ \\
\hline Age (median, range) & $61(42-72)$ \\
Sex & \\
Female & $5(18.5 \%)$ \\
Male & $22(81.5 \%)$ \\
Primary tumor location & \\
Left & $13(48.1 \%)$ \\
Right & $12(44.4 \%)$ \\
Mediastinum & $2(7.4 \%)$ \\
Type of lung tumor & \\
Central & $22(81.5 \%)$ \\
Peripheral & $5(18.5 \%)$ \\
CTNM stage & \\
IIIA & $12(44.4 \%)$ \\
IIIB & $15(55.6 \%)$ \\
Bulky mediastinal lymph node & \\
Yes & $6(22.2 \%)$ \\
No & $21(77.8 \%)$ \\
Obstructive pneumonitis/atelectasis & \\
Yes & $12(44.4 \%)$ \\
No & $15(55.6 \%)$ \\
Tumor being adjacent to esophagus & $16(59.3 \%)$ \\
No & \\
Yediastinal shift & \\
No & \\
\hline
\end{tabular}

patients (40.7\%). Mediastinal shift occurred in 3 patients (11.1\%) during the course of treatment.

\section{Gross tumor volume changes}

As shown in Fig. 2, there was significant tumor shrinkage through the course of treatment. Average GTV volume reduced from $145.1 \mathrm{~cm}^{3}$ at fraction 1 to $117.9 \mathrm{~cm}^{3}$ at fraction 17 , and the difference was statistically significant $(\mathrm{p}<0.001)$. The median relative GTV reduction was $2.1,6.2,10.6$, and $11.1 \%$ from start to fraction 5, 9, 13 and 17 of therapy, respectively. Greater tumor shrinkage was achieved from fraction 5 to 13 , compared with that from fraction 1 to 5 or fraction 13 to 17 (8.5\% vs. $2.1 \%$ vs. $0.5 \%)$. Detailed information about GTV volume change was shown in Table 2 .

\section{Dosimetric differences between original and accumulated plans}

As demonstrated in Table 3, PTV-GTV coverage were similar between original and accumulated plans. Average

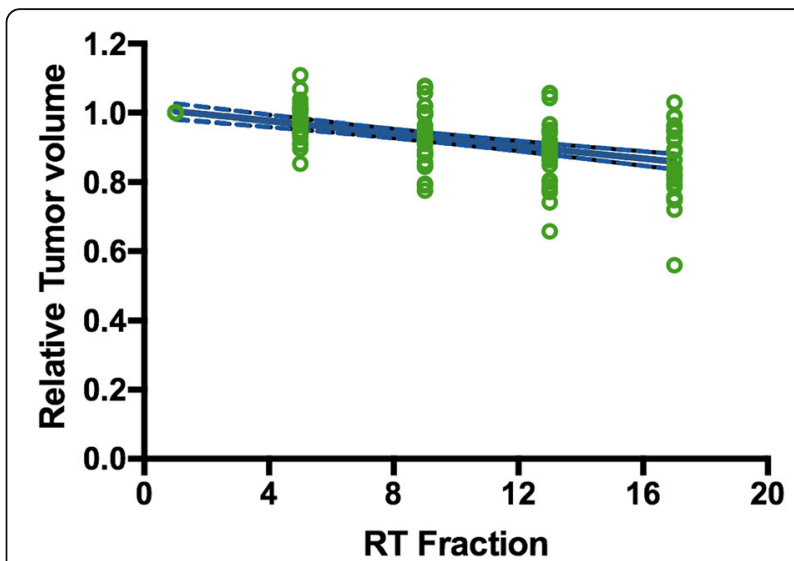

Fig. 2 The reduction in gross tumor volume during the course of radiotherapy ( $R T$ : radiotherapy)

$\mathrm{V}_{51}$ of PTV-GTV were 88.2 and $87.6 \%$ in original and accumulated plans $(p=0.452)$, with average $D_{95}$ of 48.1 Gy and $48.0 \mathrm{~Gy}(p=0.781)$, respectively. In accumulated plans, only 3 patients (11.1\%) showed a relative reduction in $\mathrm{V}_{51}$ above $5 \%$ when compared with original plans.

Besides, the $\mathrm{D}_{95}$ and $\mathrm{V}_{51}$ of PTV-GTV were compared between fraction plans (based on CBCT images at fraction $1,5,9,13$ or 17) and original plan, which was shown in Supplementary Table 1. The results indicated that the percentage of patients obtaining a reduction above $5 \%$ in $\mathrm{D}_{95}$ was higher at fraction 5 compared with that at fraction 9,13 or $17(18.5 \%$ vs. $11.1 \%$ vs. $11.1 \%$ vs. $11.1 \%, p=0.448)$; the percentage of patients obtaining a reduction above $5 \%$ in $V_{51}$ was higher at fraction 5 compared with that at fraction 9,13 or $17(22.2 \%$ vs. $14.8 \%$, $\mathrm{p}=0.448 ; 22.2 \%$ vs. $11.1 \%, p=0.278 ; 22.2 \%$ vs. $7.4 \%, p=$ $0.129)$; the percentage of patients obtaining a reduction above $5 \%$ in $\mathrm{V}_{51}$ was higher at fraction 9 compared with that at fraction 13 or $17(14.8 \%$ vs. $11.1 \%, p=0.688$; $14.8 \%$ vs. $7.4 \%, p=0.391$ ).

Slight, although statistically significant differences were observed in the lungs' dosage between original and accumulated plans. The total lung $\mathrm{V}_{20}$ increased from $29.5 \% \pm 4.2 \%$ in original plans to $30.1 \% \pm 4.1 \%$ in accumulated plans $(p=0.001)$, with a median relative increase of $1.6 \%$ (range, $-3-9 \%$ ). The total lung $\mathrm{V}_{30}$ increased from $19.9 \% \pm 4.4 \%$ in original plans to $20.4 \% \pm 4.4 \%$ in accumulated plans $(p=0.004)$, with a median relative increase of $1.9 \%$ (range, $-3-16 \%)$. Significant difference in the mean lung dose was also observed in accumulated plans $(17.0 \pm 2.1$ vs. $16.8 \pm$ $2.1, \mathrm{p}=0.001$ ), with a median relative increase of $1.4 \%$ (range, $-2-5 \%$ ). In accumulated plans, there are 3 (11.1\%) and $6(22.2 \%)$ patients obtained a relative increase above $5 \%$ in the lung $\mathrm{V}_{20}$ or $\mathrm{V}_{30}$ when compared with original plans. 
Table 2 Gross tumor volumes at different fractions of radiotherapy

\begin{tabular}{llllll}
\hline Variables & Fraction 1 & Fraction 5 & Fraction 9 & Fraction 13 & Fraction 17 \\
\hline Mean GTV $\left(\mathrm{cm}^{3}\right)$ & $145.1 \pm 159.8$ & $139.5 \pm 148.9$ & $131.2 \pm 140.2$ & $122.9 \pm 127.4$ & $117.9 \pm 122.2$ \\
Median GTV $\left(\mathrm{cm}^{3}\right)$ & $76.5(10.4-587.2)$ & $73.7(10.3-501.34)$ & $71.5(11.2-503)$ & $69.5(9.5-475.8)$ & $66.1(10.3-468.1)$ \\
Median relative GTV volume & 1.0 & $0.979(0.854-1.108)$ & $0.938(0.775-1.077)$ & $0.894(0.658-1.057)$ & $0.889(0.560-1.029)$ \\
Relative GTV Reduction & 0 & $2.1 \%(-10.8-14.6 \%)$ & $6.2 \%(-7.7-22.5 \%)$ & $10.6 \%(-5.7-34.2 \%)$ & $11.1 \%(-2.9-44 \%)$ \\
\hline
\end{tabular}

For the esophagus, an increase was observed in mean $(27.1 \pm 4.0$ vs. $26.6 \pm 4.2, p=0.002)$ and maximal doses ( $49.5 \pm 1.8$ vs. $47.4 \pm 1.3, \mathrm{p}<0.001)$ in accumulated plans, with median relative increase of $1.4 \%$ (range, -2-5\%) and $4.1 \%$ (range, $-4-15 \%$ ), respectively. Ten patients (37.0\%) obtained a relative increase above $5 \%$ in maximal dose.

There was no significant difference in the heart mean dose (13.0 \pm 6.7 vs. $13.1 \pm 7.0, p=0.558), \mathrm{V}_{30}(13.0 \pm 10.1$ vs. $12.9 \pm 10.9, p=0.675)$ or $\mathrm{V}_{40}(5.2 \pm 5.0$ vs. $4.9 \pm 5.1, p=$ 0.168 ) between original and accumulated plans. Seven patients $(25.9 \%)$ achieved above $5 \%$ relative increase in the heart $\mathrm{V}_{30}$ and 8 (29.6\%) achieved that in $\mathrm{V}_{40}$.

\section{Predictors of dosimetric differences}

Correlations between dosimetric differences and clinical variables were analyzed. Based on the magnitude of tumor shrinkage, 27 patients were divided into two groups: quick tumor regression and slow tumor regression groups. Quick tumor regression was defined as a tumor shrinkage by over $11.1 \%$ (the median value of relative GTV reduction), otherwise it was defined as slow. Patients with quick tumor regression showed more notable relative increase in the total lung $\mathrm{V}_{20}$ (median, $2.7 \%$ vs. $0.5 \%, p=0.006$ ), $\mathrm{V}_{30}$ (median, $3.5 \%$ vs. $0.2 \%, p=$ 0.022 ) and mean dose (median, $2.2 \%$ vs. $0.2 \%, p=0.009$ ) in accumulated plans than those who did not. Besides, in quick tumor regression group, more patients were observed to have a relative increase above $5 \%$ in the lung $\mathrm{V}_{20}(23.1 \%$ vs. $0, p=0.061)$ and $\mathrm{V}_{30}(38.5 \%$ vs. $7.1 \%, p=$ 0.055). Tumor regression also correlated with the differences of the esophagus mean dose $(2.2 \%$ vs. $0.2 \%, \mathrm{p}=$ $0.009)$, but not maximal dose $(4.0 \%$ vs $5.0 \%, p=0.239)$. No correlation was found between tumor regression and heart doses change (Table 4). Figure 3 shows a typical case with quick tumor regression. The doses to OARs increased markedly in accumulation plan compared with original plan.

Table 3 The comparison of dosimetric parameters between original and accumulated plans

\begin{tabular}{|c|c|c|c|c|c|}
\hline & $\begin{array}{l}\text { Original plan (mean } \pm \\
\text { SD) }\end{array}$ & $\begin{array}{l}\text { Accumulated plan (mean } \pm \\
\text { SD) }\end{array}$ & $\begin{array}{l}P \\
\text { value }\end{array}$ & $\begin{array}{l}\text { Relative change (median, } \\
\text { range) }\end{array}$ & $\begin{array}{l}\text { Above } 5 \% \text { relative reduction/ } \\
\text { increase* }\end{array}$ \\
\hline \multicolumn{6}{|l|}{ PTV-GTV } \\
\hline$V_{51}(\%)$ & $88.2 \pm 6$ & $87.6 \pm 6.3$ & 0.452 & $-0.1 \%(-10-6 \%)$ & $3(11.1 \%)$ \\
\hline$D_{95}(G y)$ & $48.1 \pm 2.0$ & $48.0 \pm 2.3$ & 0.781 & $0.1 \%(-7.6-5 \%)$ & $1(3.7 \%)$ \\
\hline $\begin{array}{l}D_{\text {mean }} \\
(\mathrm{Gy})\end{array}$ & $52.6 \pm 0.5$ & $52.6 \pm 0.6$ & 0.873 & $0.04 \%(-1-1 \%)$ & 0 \\
\hline \multicolumn{6}{|l|}{ Total lung } \\
\hline$V_{20}(\%)$ & $29.5 \pm 4.2$ & $30.1 \pm 4.1$ & 0.001 & $1.6 \%(-3-9 \%)$ & $3(11.1 \%)$ \\
\hline$V_{30}(\%)$ & $19.9 \pm 4.4$ & $20.4 \pm 4.4$ & 0.004 & $1.9 \%(-3-16 \%)$ & $6(22.2 \%)$ \\
\hline $\begin{array}{l}D_{\text {mean }} \\
(\mathrm{Gy})\end{array}$ & $16.8 \pm 2.1$ & $17.0 \pm 2.1$ & 0.001 & $1.4 \%(-2-5 \%)$ & $1(3.7 \%)$ \\
\hline \multicolumn{6}{|l|}{ Esophagus } \\
\hline $\begin{array}{l}D_{\max } \\
(\mathrm{Gy})\end{array}$ & $47.4 \pm 1.3$ & $49.5 \pm 1.8$ & $\begin{array}{l}< \\
0.001\end{array}$ & $4.1 \%(-4-15 \%)$ & $10(37.0 \%)$ \\
\hline $\begin{array}{l}D_{\text {mean }} \\
(\mathrm{Gy})\end{array}$ & $26.6 \pm 4.2$ & $27.1 \pm 4.0$ & 0.002 & $1.4 \%(-2-5 \%)$ & $1(3.7 \%)$ \\
\hline \multicolumn{6}{|l|}{ Heart } \\
\hline$V_{30}(\%)$ & $13.0 \pm 10.1$ & $12.9 \pm 10.9$ & 0.675 & $-17.4 \%(-48.6-16.4 \%)$ & 7 (25.9\%) \\
\hline$V_{40}(\%)$ & $5.2 \pm 5.0$ & $4.9 \pm 5.1$ & 0.168 & $-7.1 \%(-73.9-26.4 \%)$ & $8(29.6 \%)$ \\
\hline $\begin{array}{l}D_{\text {mean }} \\
(G y)\end{array}$ & $13.0 \pm 6.7$ & $13.1 \pm 7.0$ & 0.558 & $1 \%(-1.9-12.4 \%)$ & $5(18.5 \%)$ \\
\hline
\end{tabular}


Table 4 Dosimetric changes affected by tumor regression and baseline obstructive pneumonitis/atelectasis

\begin{tabular}{|c|c|c|c|c|}
\hline \multirow[t]{2}{*}{ Variables } & \multicolumn{2}{|l|}{ Relative change (median) \# } & \multicolumn{2}{|c|}{$\begin{array}{l}\text { Proportion of patients achieving above } 5 \% \text { reduction/ } \\
\text { increase* }\end{array}$} \\
\hline & $\begin{array}{l}\text { Tumor regression [quick }(n=13) \\
\text { vs. } \operatorname{slow}(n=14) \text { ] }\end{array}$ & $\begin{array}{l}\text { Obstructive pneumonitis/atelectasis } \\
\text { [Yes }(n=11) \text { vs. } \mathrm{No}(n=16)]\end{array}$ & $\begin{array}{l}\text { Tumor regression } \\
\text { [quick }(n=13) \text { vs. } \\
\text { slow }(n=14) \text { ] }\end{array}$ & $\begin{array}{l}\text { Obstructive pneumonitis/atelectasis } \\
\text { [Yes }(n=11) \text { vs. No }(n=16)]\end{array}$ \\
\hline \multicolumn{5}{|l|}{ PTV-GTV } \\
\hline$V_{51}$ & $0.2 \%$ vs. $-0.7 \%(P=0.185)$ & $-0.3 \%$ vs. $-0.1 \%(P=0.394)$ & $\begin{array}{l}7.7 \% \text { vs. } 14.3 \%(P= \\
0.593)\end{array}$ & $10 \%$ vs. $11.8 \%(P=0.89)$ \\
\hline$D_{95}$ & $0.3 \%$ vs. $-0.1 \%(P=0.867)$ & $-0.05 \%$ vs. $0.4 \%(P=0.942)$ & $\begin{array}{l}0 \% \text { vs. } 7.1 \%(P= \\
0.335)\end{array}$ & $10 \%$ vs. $0 \%(P=0.192)$ \\
\hline \multicolumn{5}{|l|}{ Total lung } \\
\hline$D_{\text {mean }}$ & $2.2 \%$ vs. $0.2 \%(P=0.009)$ & $2.3 \%$ vs. $0.4 \%(P=0.023)$ & $\begin{array}{l}7.7 \% \text { vs. } 0 \%(P= \\
0.299)\end{array}$ & $9.1 \%$ vs. $0 \%(P=0.228)$ \\
\hline$V_{20}$ & $2.7 \%$ vs. $0.5 \%(P=0.006)$ & $3.3 \%$ vs. $0.9 \%(P=0.110)$ & $\begin{array}{l}23.1 \% \text { vs. } O(P= \\
0.061)\end{array}$ & $9.1 \%$ vs. $12.5 \%(P=0.786)$ \\
\hline$V_{30}$ & $3.5 \%$ vs. $0.2 \%(P=0.022)$ & $3.9 \%$ vs. $0.2 \%(P=0.039)$ & $\begin{array}{l}38.5 \% \text { vs. } 7.1 \%(P= \\
0.055)\end{array}$ & $36.4 \%$ vs. $12.5 \%(P=0.150)$ \\
\hline \multicolumn{5}{|l|}{ Esophagus } \\
\hline$D_{\text {mean }}$ & $2.2 \%$ vs. $0.2 \%(P=0.009)$ & $2.3 \%$ vs. $0.4 \%(P=0.023)$ & $\begin{array}{l}7.7 \% \text { vs. } 0 \%(P= \\
0.299)\end{array}$ & $9.1 \%$ vs. $0 \%(P=0.228)$ \\
\hline$D_{\max }$ & $4.0 \%$ vs $5.0 \%(P=0.239)$ & $3.2 \%$ vs. $4.8 \%(P=0.272)$ & $\begin{array}{l}23.1 \% \text { vs. } 50 \%(P= \\
0.155)\end{array}$ & $27.3 \%$ vs. $43.8 \%(P=0.393)$ \\
\hline \multicolumn{5}{|l|}{ Heart } \\
\hline$D_{\text {mean }}$ & $0.1 \%$ vs. $0.8 \%(P=0.488)$ & $3.9 \%$ vs. $0.2 \%(P=0.251)$ & $\begin{array}{l}38.5 \% \text { vs. } \\
0 \%(P=0.012)\end{array}$ & $36.4 \%$ vs. $6.3 \%(P=0.046)$ \\
\hline$V_{30}$ & $-5.2 \%$ vs. $-1.7 \%(P=0.375)$ & $8.1 \%$ vs. $-5.2 \%(P=0.162)$ & $\begin{array}{l}38.5 \% \text { vs. } \\
14.3 \%(P=0.16)\end{array}$ & $54.5 \%$ vs. $6.3 \%(P=0.006)$ \\
\hline$V_{40}$ & $-16.1 \%$ vs. $-5.7 \%(P=0.458)$ & $2.1 \%$ vs. $-16.1 \%(P=0.272)$ & $\begin{array}{l}30.8 \% \text { vs. } \\
28.6 \%(P=0.902)\end{array}$ & $45.5 \%$ vs. $18.8 \%(P=0.143)$ \\
\hline
\end{tabular}

\#Relative change $=($ Accumulated plan-Original plan $) /$ Original plan $\times 100 \%$

*proportion of patients who had above $5 \%$ reduction in PTV-GTV dosage or above $5 \%$ increase in OARs dosage

In patients with baseline obstructive pneumonitis/atelectasis, significantly greater relative increase in the total lung mean dose (median, $2.3 \%$ vs. $0.4 \%, p=0.023$ ), lung $\mathrm{V}_{30}$ (median, $3.9 \%$ vs. $0.2 \%, p=0.039$ ) and esophagus mean dose (median, $2.3 \%$ vs. $0.4 \%, \mathrm{p}=0.023$ ) was observed compared to those without. Greater increase was also observed in the total lung $\mathrm{V}_{20}$ (median, $3.3 \%$ vs. $0.9 \%, p=0.110$ ), heart mean dose (median, $3.9 \%$ vs. $0.2 \%, p=0.251$ ), heart $\mathrm{V}_{30}$ (median, $8.1 \%$ vs. $-5.2 \%, p=$ 0.162 ) and heart $\mathrm{V}_{40}$ (median, $2.1 \%$ vs. $-16.1 \%, p=$ 0.272 ), despite none of them was statistically significant. Furthermore, in the group with baseline obstructive pneumonitis/atelectasis, more patients were observed to gain above $5 \%$ relative increase in heart $\mathrm{V}_{30}(54.5 \%$ vs. $6.3 \%, p=0.006)$ and heart mean dose $(36.4 \%$ vs. $6.3 \%$, $p=0.046)$ (Table 4).

Mediastinal shift to the ipsilateral side during treatment was observed in two cases with baseline obstructive pneumonitis. Figure 4 showed a typical case with mediastinum shifting to the ipsilateral side as tumor regressed. Increased doses to normal structures were observed, including total lung, esophagus and heart. On the contrary, mediastinal shift to the contralateral side during treatment was observed in a case with baseline obstructive atelectasis (Fig. 5). A notable decrease of $10.3 \%$ were observed in $\mathrm{V}_{51}$ of PTV-GTV. As for normal structures, decrease was also obtained in doses to total lung, esophagus and heart.

None of other clinical variables, including sex, age, tumor location, presence of bulky mediastinal lymph node or TNM stage, was found to be predictive of the dose differences of PTV-GTV or OARs between original and accumulative plans.

\section{Discussion}

Previous studies showed that HRT with a dedicated technique allows safety dose escalation, minimizing the effect of tumor repopulation and improving local control [16-18]. Compared to SBRT, a more fractionated approach was applied in modestly HRT for LANSCLC, and it mostly ranged from 12 to 20 fractions [19-21]. In this study, we accumulated the delivered dose using CBCT and DIR in patients without ART, and compared the delivered dose to planned dose. Our results indicated that, 

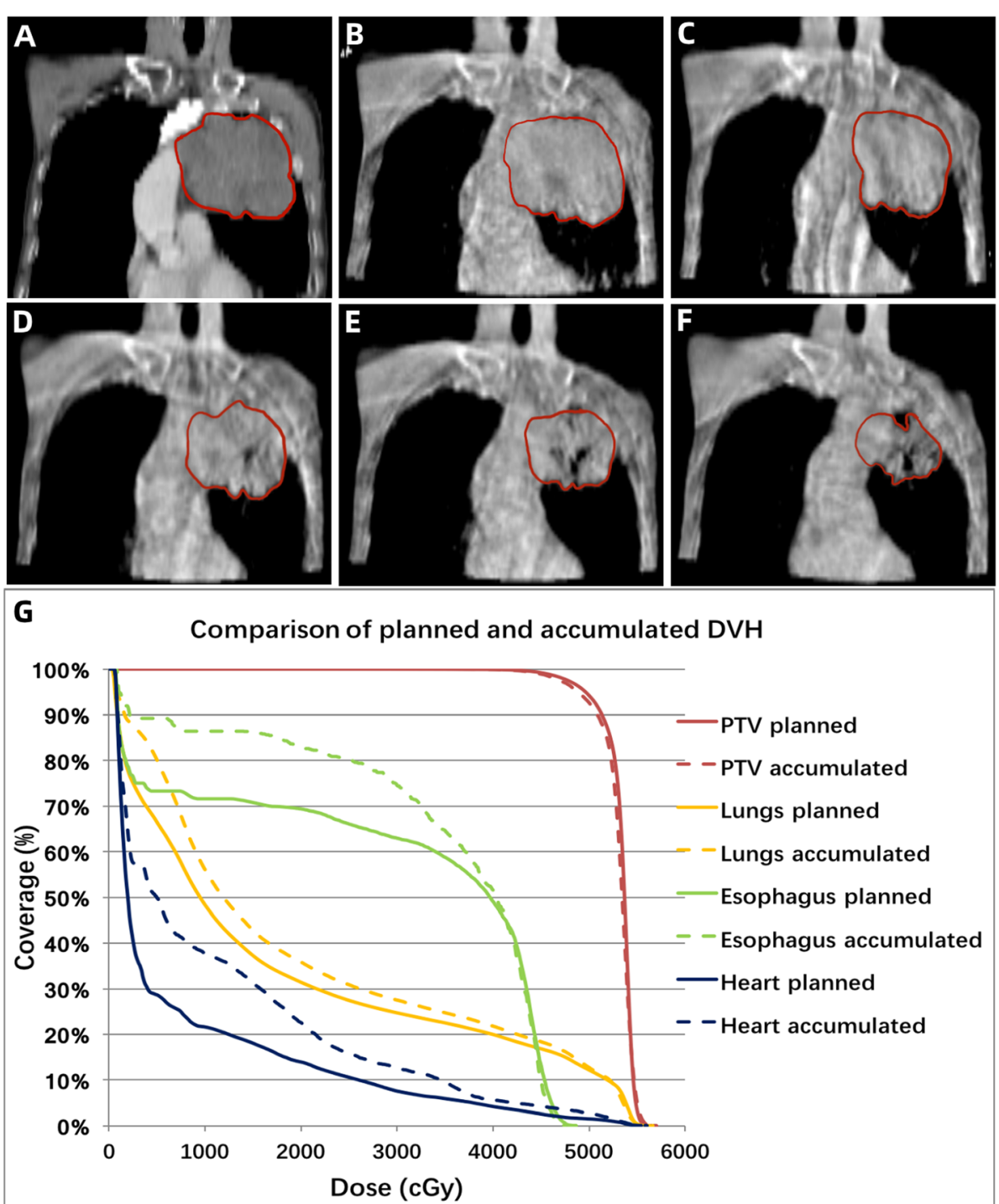

Fig. 3 Example case with quick tumor regression during radiotherapy a-f and the comparison of dose volume histogram (DVH) between original and accumulated plan (G; Solid line: original plan, Dashed line: accumulated plan). The GTV volume was estimated in contrast-enhanced simulation $C T \mathbf{a}$, and CBCT at fraction $1 \mathbf{b}$, fraction $5 \mathbf{c}$, fraction $9 \mathbf{d}$, fraction $13 \mathbf{e}$ and fraction $17 \mathbf{f}$ of radiotherapy

for most patients, the differences between delivered and planned dose were clinically acceptable. The median relative reduction in $\mathrm{V}_{51}$ of PTV-GTV was only $0.1 \%$. In terms of normal tissues, although statistically significant difference was observed in lung and esophagus doses between original and accumulated plans, the median relative increase in most metrics was very limited (less than $2 \%$ ), except for maximal esophagus dose with a median relative increase of $4.1 \%$. Considering that a $5 \%$ relative difference in dosimetric parameters would be clinically meaningful, we analyzed the number of patients who obtained above 5\% relative reduction in PTV-GTV coverage or $5 \%$ relative increase in normal organs' dosage. Overall, only $11.1 \%$ of patients were observed to have above $5 \%$ relative decrease in $\mathrm{V}_{51}$ of PTV-GTV.
Meanwhile, less than $30 \%$ of patients achieved above $5 \%$ relative increase in lung or heart doses. According the results, non-ART strategy was acceptable for most patients treated by HRT, and only a small proportion of patients might be in need of ART during the treatment.

For these reasons, the changes of GTV during treatment course need to be investigated. Previous studies found that the mean GTV reduction was about 19$51.1 \%$ in patients treated by conventional fractionated radiotherapy [22-25]. A less marked reduction in tumor volume was obtained during SBRT due to the short irradiation course, with the mean GTV reduction of $12.8-$ $32 \%[26,27]$. In current study, we detected a gradual shrinking of tumor volume over the course of treatment with median reduction of $11.1 \%$, which suggested only a 


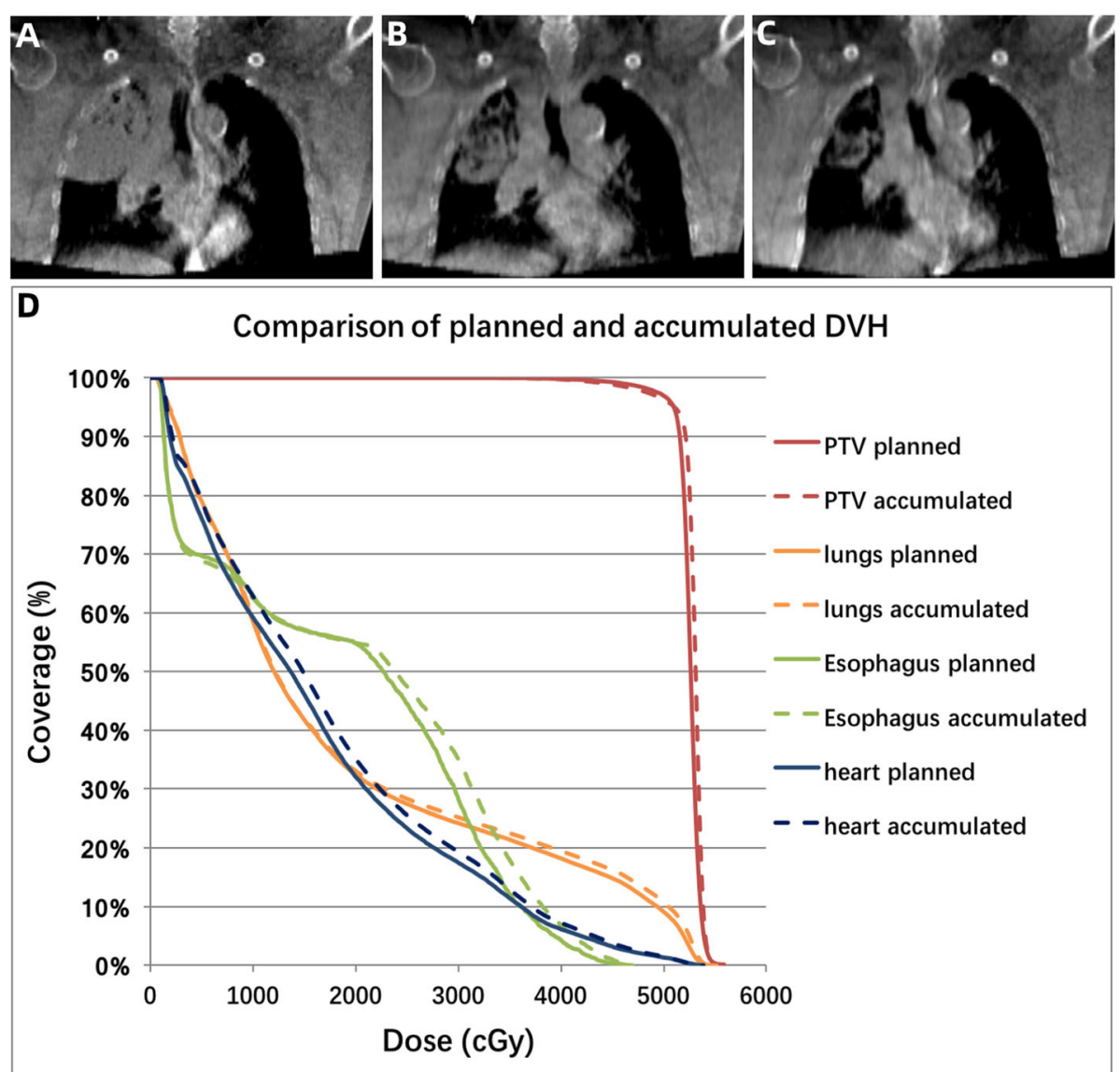

Fig. 4 A typical case with mediastinum shifting to the ipsilateral side as tumor regressed. a CBCT scan performed at fraction 1 of radiotherapy; $\mathbf{b}$ c CBCT scans at fraction 9 and 17 revealed a mediastinal shift to the ipsilateral side; $\mathbf{d}$ the comparison of dose volume histogram (DVH) between original and accumulated plan (Solid line: original plan, Dashed line: accumulated plan)

fraction of patients could benefit from ART. The extent of tumor regression has been used to identify candidates for ART in previous studies [28, 29]. Woodford et al. found that ART was required when a patient had a greater than $30 \%$ reduction in tumor volume within the first 20 fractions [28]. Therefore, we identified the correlation between tumor regression and the dosimetric difference. Patients with GTV reduction over $11.1 \%$ had more significant increase in lung and esophagus doses in the accumulated plans. It supported the idea that the magnitude of GTV reduction might be used as an indicator for plan adaption. Previous study explored the optimal time point for plan adaption among NSCLC treated by modestly HRT (10-12 fractions). And the treatment midpoint was considered as a favorable time for plan adaptation, since notable tumor response and sufficient remaining fractions were both obtained at present [29]. Similarly, in our study, notable tumor response was achieved from fraction 5 to 13 . In order to further identify the optimal time point in the course of radiotherapy for re-planning, we analyzed the number of patients who obtained a reduction above $5 \%$ in $\mathrm{D}_{95}$ and
$\mathrm{V}_{51}$ of PTV-GTV when comparing the fraction plan at different time point of treatment course with the original plan. The results showed that the percentage of patients obtaining a reduction above $5 \%$ in $\mathrm{D}_{95}$ and $\mathrm{V}_{51}$ of PTVGTV were higher at fraction 5 compared with that at fraction 9,13 , or 17 . Besides, a higher percentage of patients obtaining $>5 \%$ reduction in $\mathrm{V}_{51}$ of PTV-GTV was also observed at fraction 9 compared with that at fraction 13 or 17. Taking together the changes in GTV volume, $D_{95}$ and $V_{51}$ of PTV-GTV at different time points, we recommended the fraction 9 in the course of radiotherapy as the time point for re-planning.

For lung cancer patients with obstructive pneumonitis or atelectasis before radiotherapy, the volume and position of lung tissue could progressively change with treatment [30]. Previous studies reported that the resolution rate of atelectasis was $38-90 \%$ with thoracic radiotherapy in lung cancer patients [31-33]. Great deviation in dose distribution might occur when a patient experiences great changes in the lung tissue. Moller et al. found that ART was required in $70 \%$ of lung cancer patients with atelectasis [34]. Our study indicated 

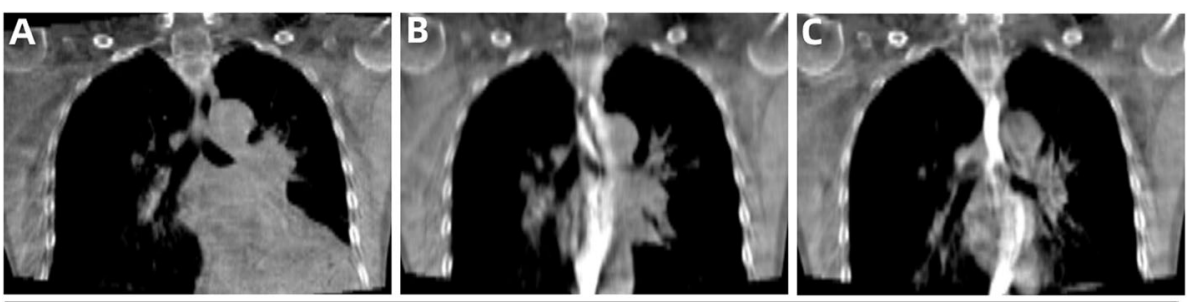

D

Comparison of planned and accumulated DVH

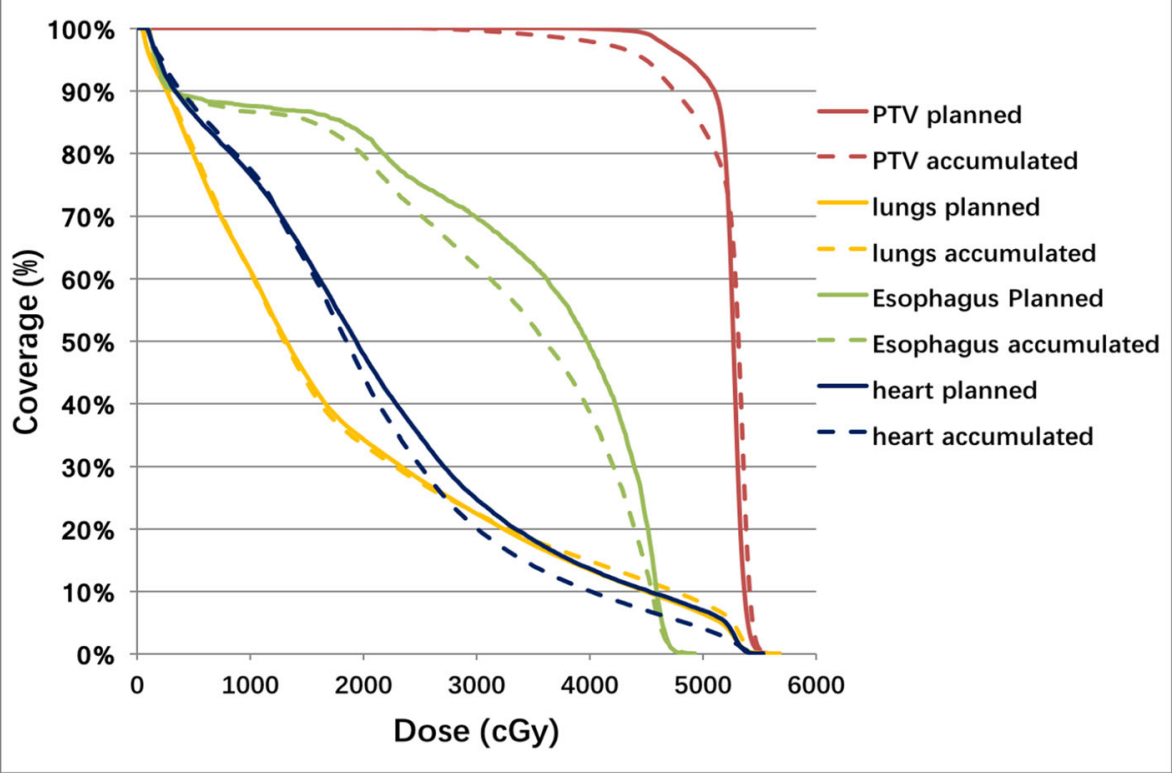

Fig. 5 A typical case with mediastinum shifting to contralateral side as lung re-expanded. a CBCT scan performed at fraction 1 of radiotherapy; bc. CBCT scans at fraction 9 and 17 revealed a mediastinal shift to the contralateral side; $\mathbf{d}$ the comparison of dose volume histogram (DVH) between original and accumulated plan (Solid line: original plan, Dashed line: accumulated plan)

that patients with baseline obstructive pneumonitis/atelectasis achieved more notable increase in total lung, esophagus and heart dose than those without. In addition, 54.5 and $45.5 \%$ of patients with obstructive pneumonitis/atelectasis were observed to gain above $5 \%$ relative increase in heart V30 and V40, respectively. The results suggested that patients with baseline obstructive pneumonitis or atelectasis might benefit more from ART. And frequent CBCT scans should be performed in order to enable the immediate access to ART when great change in lung volume and position was obtained.

Other than the change of lung volume and position, mediastinal shift remained crucial for dose deviation of OARs for patients with baseline obstructive pneumonitis/atelectasis. Mediastinal shift could occur as the regression of tumor and resolution of pneumonitis/ atelectasis during radiotherapy. In fact, it is difficult to directly quantify the movement of mediastinum, since it consisted of multiple organs and structures. Esophagus, as a longitudinal organ located in the middle of mediastinum, could largely reflect the motion of mediastinum.
In previous study, we quantified the interfractional motion of esophagus during fractionated radiotherapy for 36 patients with locally advanced non-small cell lung cancer [35]. Oral barium sulfate was administrated during CBCT to help localize the esophagus. The results indicated that the motion in right-left direction were $24.0 \sim 11.9 \mathrm{~mm}, \quad-27.8 \sim 13.1 \mathrm{~mm}$ and $-36.3 \sim 10.4 \mathrm{~mm}$ for proximal, middle and distal thoracic esophagus respectively. These data could to some extent reflect the movement of mediastinum. In the present study, the presence of upper and middle mediastinum shift was defined as a right-left motion of esophagus above $10 \mathrm{~mm}$, which had occurred in three patients. The occurrence of mediastinal shift might result in inadequate tumor dose coverage or increased normal tissue doses [36]. In patients with baseline obstructive pneumonitis, the mediastinum usually deviated to the ipsilateral side with the remission of tumor, which resulted in the increased exposure of mediastinal structures. However, for patients with baseline obstructive atelectasis, the mediastinum might deviate to the contralateral side because of the re- 
expansion of lung after treatment. The inadequate dose coverage of target volume remained the main concern for these patients. Despite the example size was limited, we still supposed that the plan adaption should be taken into account for patients with baseline obstructive atelectasis and mediastinal shift.

The limitation of the study is that the dose accumulation was not based on single delivered dose of each fraction, which may have an impact on the accuracy of dose accumulation. We chose fractionated dose at five timepoints (fraction 1, 5, 9, 13 and 17) for dose calculation and accumulation, which can largely reflect the actual dose that patient has received. Tissue appearance and disappearance (TAD) (such as the tumor regression) might also hamper the accuracy of DIR between CBCT and reference CT [37]. While it was still an open problem to be addressed by the radiotherapy community, measures had been taken to minimize the impact of TAD and lung tissue changes on the DIR accuracy. With careful inspection of the DIR results using anatomical landmarks, dose accumulation uncertainties could be reduced to the clinical acceptable level [38].

\section{Conclusions}

In summary, most patients with LANSCLC had sufficient tumor dose coverage and acceptable normal tissue dose deviation without ART in the treatment of modestly HRT. Quick tumor regression, baseline obstructive pneumonitis/atelectasis during treatment are potential indicators for the need of ART. Daily CBCT scans prior to treatment was essential for patients treated by HRT, which enabled the timely detection of anatomical change for tumors and normal structures. To assess the advantage of ART based on the above factors in prospective studies are warranted.

\section{Supplementary Information}

Supplementary information accompanies this paper at https://doi.org/10 1186/s12885-020-07617-3.

Additional file 1: Supplementary table 1. The comparison of PTVGTV $D_{95}$ and $V_{51}$ between the fraction plans at different time point of treatment course with the original plan.

\section{Abbreviations}

LANSCLC: Locally advanced non-small cell lung cancer;

HRT: Hypofractionated radiotherapy; OAR: Organs at risk; SIB-

IMRT: Simultaneous integrated boost intensity-modulated radiation therapy; ART: Adaptive radiotherapy; CBCT: Cone beam CT; DIR: Deformable image registration; $4 D C T$ : Four-dimensional CT; MIP: Maximum intensity projection; DVF: Deformation vector field; TPS: Treatment planning system; GTV: Gross tumor volume; CTV: Clinical target volume; PTV: Planning target volume; DVH: Dose volume histogram

\section{Authors' contributions}

All authors should have made substantial contributions to all of the following:

(1) $B Q, H L, B W$ contributed to the conception and design of the study. (2) BW, BQ, HL, DQW, MSL, SPL, JZ, LC, QWL, ZKC, FJL and JYG contributed to the acquisition of image and data. (3) DQW, BW, BQ, HL, MSL, SPL, JZ, LC, QWL, ZKC, FJL and JYG analyzed and interpreted data. (4) DQW and BQ drafted the article. (5) $B Q, H L, B W$ and DQW critically revised the manuscript. All authors read and approved the submitted version.

\section{Funding}

This work was supported by Science and Technology Planning Project of Guangdong Province, China [Grant Number 2016A020215190]. The grant covered the costs of image acquisition, image analysis and data storage required for the study.

\section{Availability of data and materials}

The datasets used and/or analyzed during the current study are available from the corresponding author on reasonable request.

\section{Ethics approval and consent to participate}

This study was approved by the Ethical Committee of GuangDong Association Study of Thoracic Oncology (GASTO), and our team has acquired the administrative permissions to access the clinical patient data used in this research from GASTO (Grant No. B2019-075). All participants provide written informed consent.

\section{Consent for publication}

Not applicable.

\section{Competing interests}

The authors declare that they have no competing interests.

Received: 12 August 2020 Accepted: 5 November 2020

Published online: 16 November 2020

\section{References}

1. Bezjak A, Temin S, Franklin G, et al. Definitive and adjuvant radiotherapy in locally advanced non-small-cell lung Cancer: American Society of Clinical Oncology clinical practice guideline endorsement of the American Society for Radiation Oncology evidence-based clinical practice guideline. J Clin Oncol. 2015:33:2100-5.

2. Postmus PE, Kerr KM, Oudkerk $M$ et al. Early and locally advanced nonsmall-cell lung cancer (NSCLC): ESMO Clinical Practice Guidelines for diagnosis, treatment and follow-up. Ann Oncol 2017; 28: iv1-iv21.

3. Qiu B, Li QW, Ai XL, et al. Investigating the loco-regional control of simultaneous integrated boost intensity-modulated radiotherapy with different radiation fraction sizes for locally advanced non-small-cell lung cancer: clinical outcomes and the application of an extended LQ/TCP model. Radiat Oncol. 2020;15:124.

4. Fakir H, Chen J, Sachs RK. Hypo-fractionated boost in locally advanced nonsmall cell lung cancer: temporal distribution of boost fractions. Phys Med Biol. 2018;63:235018.

5. Korreman SS. Motion in radiotherapy: photon therapy. Phys Med Biol. 2012; 57:R161-91.

6. Schwarz M, Cattaneo GM, Marrazzo L. Geometrical and dosimetrical uncertainties in hypofractionated radiotherapy of the lung: a review. Phys Med. 2017;36:126-39.

7. Jaffray DA, Siewerdsen JH, Wong JW, Martinez AA. Flat-panel cone-beam computed tomography for image-guided radiation therapy. Int J Radiat Oncol Biol Phys. 2002;53:1337-49.

8. Kurz C, Kamp F, Park YK, et al. Investigating deformable image registration and scatter correction for CBCT-based dose calculation in adaptive IMPT. Med Phys. 2016;43:5635

9. Chetty IJ, Rosu-Bubulac M. Deformable registration for dose accumulation Semin Radiat Oncol. 2019;29:198-208.

10. Flampouri S, Jiang SB, Sharp GC, et al. Estimation of the delivered patient dose in lung IMRT treatment based on deformable registration of 4D-CT data and Monte Carlo simulations. Phys Med Biol. 2006;51:2763-79. 
11. Hugo GD, Campbell J, Zhang T, Yan D. Cumulative lung dose for several motion management strategies as a function of pretreatment patient parameters. Int J Radiat Oncol Biol Phys. 2009;74:593-601.

12. Dial C, Weiss E, Siebers JV, Hugo GD. Benefits of adaptive radiation therapy in lung cancer as a function of replanning frequency. Med Phys. 2016;43:1787.

13. Cole AJ, Veiga $C$, Johnson $U$, et al. Toward adaptive radiotherapy for lung patients: feasibility study on deforming planning CT to CBCT to assess the impact of anatomical changes on dosimetry. Phys Med Biol. 2018;63:155014.

14. Ma L, Qiu B, Li Q, et al. An esophagus-sparing technique to limit radiation esophagitis in locally advanced non-small cell lung cancer treated by simultaneous integrated boost intensity-modulated radiotherapy and concurrent chemotherapy. Radiat Oncol. 2018;13:130.

15. Lawson JD, Schreibmann E, Jani AB, Fox T. Quantitative evaluation of a cone-beam computed tomography-planning computed tomography deformable image registration method for adaptive radiation therapy. J Appl Clin Med Phys. 2007:8:2432.

16. Cannon DM, Mehta MP, Adkison JB, et al. Dose-limiting toxicity after hypofractionated dose-escalated radiotherapy in non-small-cell lung cancer. J Clin Oncol. 2013;31:4343-8.

17. Donato $\mathrm{V}$, Arcangeli S, Monaco A, et al. Moderately escalated Hypofractionated (chemo) radiotherapy delivered with helical intensitymodulated technique in stage III Unresectable non-small cell lung Cancer. Front Oncol. 2013;3:286.

18. Urbanic JJ, Wang X, Bogart JA, et al. Phase 1 study of accelerated Hypofractionated radiation therapy with concurrent chemotherapy for stage III non-small cell lung Cancer: CALGB 31102 (Alliance). Int J Radiat Oncol Biol Phys. 2018;101:177-85.

19. Harris JP, Chang-Halpenny CN, Maxim PG, et al. Outcomes of modestly Hypofractionated radiation for lung tumors: pre- and mid-treatment positron emission tomography-computed tomography metrics as prognostic factors. Clin Lung Cancer. 2015;16:475-85.

20. Osti MF, Agolli L, Valeriani M, et al. Image guided hypofractionated 3dimensional radiation therapy in patients with inoperable advanced stage non-small cell lung cancer. Int J Radiat Oncol Biol Phys. 2013;85:e157-63.

21. Soliman $H$, Cheung $P$, Yeung $L$, et al. Accelerated hypofractionated radiotherapy for early-stage non-small-cell lung cancer: long-term results. Int J Radiat Oncol Biol Phys. 2011;79:459-65.

22. Juhler-Nottrup T, Korreman SS, Pedersen AN, et al. Interfractional changes in tumour volume and position during entire radiotherapy courses for lung cancer with respiratory gating and image guidance. Acta Oncol. 2008;47: 1406-13.

23. Lim G, Bezjak A, Higgins J, et al. Tumor regression and positional changes in non-small cell lung cancer during radical radiotherapy. J Thorac Oncol. 2011;6:531-6.

24. Guckenberger M, Wilbert J, Richter A, et al. Potential of adaptive radiotherapy to escalate the radiation dose in combined radiochemotherapy for locally advanced non-small cell lung cancer. Int $J$ Radiat Oncol Biol Phys. 2011;79:901-8.

25. Zhong H, Siddiqui SM, Movsas B, Chetty IJ. Evaluation of adaptive treatment planning for patients with non-small cell lung cancer. Phys Med Biol. 2017; 62:4346-60.

26. Sun Y, Lu Y, Cheng S, et al. Interfractional variations of tumor centroid position and tumor regression during stereotactic body radiotherapy for lung tumor. Biomed Res Int. 2014;2014:372738.

27. Bhatt AD, El-Ghamry MN, Dunlap NE, et al. Tumor volume change with stereotactic body radiotherapy (SBRT) for early-stage lung cancer: evaluating the potential for adaptive SBRT. Am J Clin Oncol. 2015;38:41-6.

28. Woodford C, Yartsev S, Dar AR, et al. Adaptive radiotherapy planning on decreasing gross tumor volumes as seen on megavoltage computed tomography images. Int J Radiat Oncol Biol Phys. 2007;69:1316-22.

29. Henke LE, Kashani R, Hilliard J, et al. In Silico trial of MR-guided Midtreatment adaptive planning for Hypofractionated stereotactic radiation therapy in centrally located thoracic tumors. Int J Radiat Oncol Biol Phys. 2018;102:987-95

30. Sonke JJ, Belderbos J. Adaptive radiotherapy for lung cancer. Semin Radiat Oncol. 2010;20:94-106

31. Majid OA, Lee $\mathrm{S}$, Khushalani $\mathrm{S}$, Seydel HG. The response of atelectasis from lung cancer to radiation therapy. Int J Radiat Oncol Biol Phys. 1986;12:231-2.

32. Reddy SP, Marks JE. Total atelectasis of the lung secondary to malignant airway obstruction. Response to radiation therapy. Am J Clin Oncol. 1990;13: 394-400.
33. Vaaler AK, Forrester JM, Lesar M, et al. Obstructive atelectasis in patients with small cell lung cancer. Incidence and response to treatment. Chest. 1997;111:115-20.

34. Moller DS, Khalil AA, Knap MM, Hoffmann L. Adaptive radiotherapy of lung cancer patients with pleural effusion or atelectasis. Radiother Oncol. 2014; 110:517-22.

35. Qiu B, Lu S, Wang B, et al. Quantifying the Interfractional motion of esophagus using daily cone beam computed tomography with Oral contrast during radiation therapy for locally advanced non-small cell lung Cancer. Pract Radiat Oncol. 2020;10:e339-47.

36. Sakanaka K, Fujii K, Mizowaki T. Adaptive radiotherapy in locally advanced esophageal cancer with atelectasis: a case report. BMC Cancer. 2020;20:21.

37. Samavati N, Velec M, Brock KK. Effect of deformable registration uncertainty on lung SBRT dose accumulation. Med Phys. 2016;43:233.

38. Nobnop W, Chitapanarux I, Neamin H, et al. Evaluation of deformable image registration (DIR) methods for dose accumulation in nasopharyngeal Cancer patients during radiotherapy. Radiol Oncol. 2017;51:438-46.

\section{Publisher's Note}

Springer Nature remains neutral with regard to jurisdictional claims in published maps and institutional affiliations.
Ready to submit your research? Choose BMC and benefit from:

- fast, convenient online submission

- thorough peer review by experienced researchers in your field

- rapid publication on acceptance

- support for research data, including large and complex data types

- gold Open Access which fosters wider collaboration and increased citations

- maximum visibility for your research: over $100 \mathrm{M}$ website views per year

At BMC, research is always in progress.

Learn more biomedcentral.com/submissions 